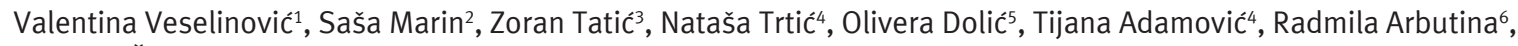
Miodrag Šćepanović ${ }^{7}$, Aleksandar Todorović $\tilde{c}^{7}$

\title{
Application of Semipermanent Cements and Conventional Cement with Modified Cementing Technique in Dental Implantology
}

\section{Primjena polutrajnih i konvencionalnih cemenata s modificiranom tehnikom cementiranja u dentalnoj implantologiji}

\footnotetext{
${ }^{1}$ Department of Prosthodontics, Faculty of Medicine, University of Banja Luka, Bosnia and Herzegovina Katedra za protetiku Medicinskog fakulteta Univerziteta u Banjoj Luci, Bosna i Hercegovina

2 Department of Oral Surgery, Faculty of Medicine, University of Banja Luka, Bosnia and Herzegovina Katedra za oralnu kirurgiju Medicinskog fakulteta Univerziteta u Banjoj Luci, Bosna i Hercegovina

${ }^{3}$ Clinic of Dental Medicine, Military Medical Academy, Belgrade, Serbia

Klinika za dentalnu medicinu Vojne medicinske akademije, Beograd, Srbija

${ }^{4}$ Department of Periodontology and Oral Medicine, Faculty of Medicine, University of Banja Luka, Bosnia and Herzegovina Katedra za parodontologiju i oralnu medicinu Medicinskog fakulteta Univerziteta u Banjoj Luci, Bosna i Hercegovina

${ }^{5}$ Department of Pediatric and Preventive Dentistry, Faculty of Medicine, University of Banja Luka, Bosnia and Herzegovina Katedra za dječju i preventivnu stomatologiju Medicinskog fakulteta Univerziteta u Banjoj Luci, Bosna i Hercegovina

${ }^{6}$ Department of Restorative Dentistry, Faculty of Medicine, University of Banja Luka, Bosnia and Herzegovina Katedra za restaurativnu stomatologiju Medicinskog fakulteta Univerziteta u Banjoj Luci, Bosna i Hercegovina

7 Department of Prosthodontics, School of Dental Medicine, University of Belgrade, Serbia

Klinika za stomatološku protetiku, Stomatološki fakultet, Univerzitet u Beogradu, Srbija
}

\section{Abstract}

Objectives: The aim of this study was to evaluate the influence of artificial ageing on the retention force of original semipermanent cements, as well as the possibility of using conventional cements for semipermanent cementation with adequate modification of the cementing protocol. Material and methods: Forty CoCrMo alloy crowns were divided in four groups (each group $n=10$ ) and fixed with two semipermanent cements (resin-based and glass ionomer-based cements) and one conventional (zinc phosphate), using conventional and modified cementation techniques on titanium abutments. The samples were stored in humid conditions for 24 hours at $37^{\circ} \mathrm{C}$ and subjected to thermocycling (500 cycles) and mechanical cyclic loading (7 days, 3, 6, 9 and 12 months function simulation). The cast crowns were removed and the retention force was recorded. Results: The highest initial retention force measured was for zinc-phosphate cement - conventional cementing $(198,00 \pm 61,90 \mathrm{~N})$, followed in descending order by zinc-phosphate cement - modified cementing technique $(152,00 \pm 45,42$ $\mathrm{N})$, long term temporary cement - GC Fuji Temp LT (57,70 $\pm 20,40 \mathrm{~N})$, and semipermanent cement - Telio CS Cem Implant $(56,10 \pm 18,68 \mathrm{~N})$. After 12 months, the highest retention force measured was for zinc-phosphate cement - conventional cementing $(88,90 \pm 14,45 \mathrm{~N})$, followed by zinc-phosphate cement - modified cementing $(48,15 \pm 14,41 \mathrm{~N})$, semipermanent cement GC Fuji Temp LT $(16,55 \pm 3,88 \mathrm{~N})$ and Telio CS Cem Implant (15,55 $\pm 5,52 \mathrm{~N})$. Conclusions: Zinc-phosphate cement - modified cementing technique and original semipermanent cements can be recommended for conditional permanent cementing of implant supported crowns. Clinical relevance: The use of semipermanenet cements and zinc-phosphate cement - modified cementing technique provides a predictable retrievability of implant-supported crowns.
Received: May 19, 2021

Accepted: September 3, 2021

Address for correspondence Associate Professor Valentina Veselinović, PhD, DDM University of Banja Luka Faculty of Medicine Department of Prosthodontics Save Mrkalja 14, 78000 Banja Luka, Bosnia and Herzegovina Phone: +38751234100 valentina.veselinovic@med.unibl.org

MeSH terms: Dental Cements; Mechanical Tests; Cementation; Dental Implantation

Author keywords: Crown Retrievability; Semipermanent Cements; Conventional Cements; Artificial Ageing; Retention Force 


\section{Introduction}

The long-term success of implantoprosthetic therapy depends on several factors: good osseointegration of the implant, the quality of the prosthetic reconstruction, and the connection between the implant and a crown. Due to the specific connection between the implant and bone in implantology, there is a specific transfer of loading. This connection is rigid, with no shock absorber mechanism for masticatory force. This has to be taken into consideration throughout the entire prosthetic planning and treatment.

The most commonly used retention modes are cementation and screw-retention. The professional community is divided regarding the use of cement or fixation screws for implant supported crown fixation. In fact, there has been controversy about whether to use cement-retained or screwsretained implant supported crowns, mostly based on retrievability versus esthetics (1-4).

Passivity is another extremely important factor when analyzing the features of implant-supported crowns. The analysis of the currently available scientific studies showed that, complete passivity of screw-retained implant supported crowns is still a challenge and it should be a goal of modern implantology $(5,6)$. On the other hand, dental cements allow the crown to sit passively on implant abutment, filling the space between abutment and crown, hence enabling the compensation of small non-concurrences in a crown fitting (7-9).

A wide variety of dental cements, with different properties, are commercially available to retain an implant-supported prosthesis. Retention and esthetics are the two main factors to consider when choosing which cement selection guidelines are best for individual patients (10).

The retrievability of cemented crowns is highly limited. Removing permanently cemented crowns from abutment requires significant force. This might result in permanent damage of the crown, abutment and even the implant itself. On the other hand, temporary cements might allow easy crown removal, but, taking into consideration their low retention level, marginal leakage, and dissolubility in oral fluids, these cements cannot provide adequate retention and long-term stability of a crown. Ideally, cement should be strong enough to retain the crown, and at the same time, sufficiently weak to allow the dentist to remove the crown when needed. The level of force required to remove the crown should not cause any implant trauma and damage to the crown and the abutment (11).

Compared to natural teeth and their adaptation mechanisms, implants have no periodontal ligament support (12), thereby lacking adequate shock absorption and adaptability to the high short-term forces which are required during crown removal. Thus, compared to natural teeth, crown removal might represent an implant overloading (11).

There is a need for a solution that would enable adequately retained crown and bridges with the simultaneous possibility of retrievability when necessary. The first attempts to weaken retention and try to increase the ease of retrievability were by using petroleum jelly in addition to permanent cements ("Hand-made cements") (13).
Uvod

Uspjeh implantoprotetičke terapije, gledano na višegodišnjoj razini, ovisi o više čimbenika - o dobroj oseointegraciji implantata, kvalitetno izrađenoj protetičkoj nadoknadi i vezi između implantata i same nadoknade. U implantologiji, zbog posebne veze implantata i kosti, specifičan je prijenos pritiska. Ta je veza kruta, bez mehanizma amortizacije žvačnih (mastikatornih) sila. To je činjenica koja se tijekom planiranja i izrade nadoknade ne smije zanemariti.

Najčešćc fiksacije su cementiranje i pričvršćivanje vijcima. Fiksiranje zubne nadoknade na nosač nadoknade cementom ili fiksacijskim vijkom podijelilo je stručnu javnost. Mišljenja se razlikuju kad je riječ o upotrebi cemenata prema krunicama na nadoknadama fiksiranima vijcima i u velikoj se mjeri nastavljaju rasprave o mogućnosti njihova uklanjanja u usporedbi s estetikom $(1-4)$.

Pasivnost je sljedeći itekako važan čimbenik kada analiziramo karakteristike krunica postavljenih na implantate. Analize trenutačno dostupnih znanstvenih članaka pokazale su da je potpuna pasivnost restauracija fiksiranih vijcima na implantatima-nosačima još uvijek izazov, a treba biti cilj suvremene implantologije (5, 6). S druge strane, dentalni cementi omogućuju pasivno ležanje nadoknade na implantatu-nosaču, ispunjavajući prostor između nadogradnje i krunice te omogućujući kompenzaciju malih nepreciznosti pri nalijeganju $(7-9)$.

$\mathrm{Na}$ tržišstu je velik broj dentalnih cemenata različitih karakteristika za retenciju nadoknada na implantatima. Retencija i estetika dva su osnovna čimbenika koja se uzimaju u obzir pri selekcijskim kriterijima za izbor najboljeg cementa za svakog pacijenta (10).

Mogućnost uklanjanja nadogradnji retiniranih cementima visoko je limitirana. Uklanjanje trajno cementirane krunice $s$ nadogradnje zahtijeva primjenu dosta velike sile. Tim se postupkom mogu trajno oštetiti krunica, nadogradnja pa čak i implantat. $S$ druge strane, privremeni cementi omogućuju lagano uklanjanje nadoknade, ali ako uzmemo u obzir nizak stupanj retencije, marginalno propuštanje i topivost u oralnim tekućinama, ti cementi ne mogu dulje osigurati odgovarajuću retenciju i stabilnost nadoknade u funkciji.

Cement bi trebao biti dovoljno jak da retinira krunicu, a istodobno dovoljno slab da omogući doktoru da je ukloni bude li to potrebno. Razina sile potrebne za uklanjanje nadoknade mora biti tolika da ne prouzroči bilo kakvu traumu implantata te oštećenje krunice i nadogradnje (11).

U usporedbi s prirodnim zubima i njihovim mehanizmima adaptacije, implantati nemaju potporu okolnih ligamenata (12), što rezultira nedostatkom adekvatnoga mehanizma amortizacije sile i adaptacije na kratkotrajne sile visokog intenziteta koje je potrebno primijeniti pri pokušaju skidanja nadoknade. Zato u usporedbi s prirodnim zubom, uklanjanje krunica postavljenih na implantate može preopteretiti implantat (11). Potrebno je pronaći rješenje koje će omogućiti adekvatnu retenciju krunica i mostova, uz istodobnu mogućnost njihova uklanjanja bude li potrebno. U prvim pokušajima da se oslabi retencija cementiranjem i poveća mogućnost njihova uklanjanja dodavao im se vazelin (hand-made cements) (13). 
However, due to the arbitrary retention obtained, this method was soon abandoned. Another way to reach an ideal balance is to apply conventional cement (zinc-phosphate or conventional glass ionomer cement) only on the crown's edges. In this case, the unfavorable characteristic of marginal dissolubility and the level of discrepancy of conventional cements can result in a decrease of cement retention after a certain period of time $(10,14,15)$.

There are also specifically designed cements for long-term temporary cementing which enable adequate crown retention for the period of 6 months to one year. This semipermanent cementation model and retrievability modalities have been reviewed in some studies (16-22).

In vitro conditions may be used to simulate some influential factors of the oral environment and material's potential performance in vivo. One of the conventionally used systems of artificial ageing is thermal cycling which includes subjecting of samples to repeated cycle of hot and cold temperatures, in order to reproduce thermal changes occurring in the oral cavity (23). Thermal changes induce stress in dental materials; therefore the properties of materials could be changed.

Compressive cyclic loading represents a method of simulating occlusal stresses encountered in the oral environment. The mentioned method of masticatory cycle's simulation was used in the observation of dental cements retention force in different time points $(24,25)$.

Hence, the aim of this study was to test the influence of artificial ageing on the retention force of semipermanent cements, as well as the possibility of using conventional cements for semipermanent cementing with a specific modification of the cementing protocol.

The following hypothesis has been tested:

There is no difference in the retention provided by all tested cements - GC Fuji Temp LT, Telio CS Cem Implant and Harvard Cement (standard and modified cementing technique) at retention measurement time-points.

There is no influence of thermal cycling and compressive cyclic loading on the physical retention of tested cements at measurement time points.

\section{Material and methods}

The working model was 40 titanium abutments (Easy abutment NP 0,75, Nobel Biocare, Sweden), conical by $8^{\circ}$, height 5,5 mm, 40 implant replicas (Implant Replica, Nobel Biocare, Sweden. Abutments were screwed into implant replicas with $35 \mathrm{~N} / \mathrm{cm}$ of torque. The crowns made from $\mathrm{CoCr}-$ Mo alloy (Bond NF - Nickel free, Interdent, Slovenia) were used (Figure 1, 2).

The abutment access channel was closed with a temporary light polymerizing composite (Temp it, Spident, South Korea). In this study, the crowns were made based on a silicon mold with occlusal two-thirds of the crown of acrylate Frasaco tooth - second lower premolar. The inner side of the crown and the thickness of the cemented space were standardized by using $7 \mathrm{~mm}$ plastic molding caps (Plastic Coping Easy Abutment Engaging NP 2 pkg). After the casting, the outer surface of the crown was highly-polished, whereas
Tako dobivena retencija bila je proizvoljna i izvan kontrole te se ubrzo odustalo od takvog postupka. Drugi način, korišten u svrhu idealnog balansa, jest nanošenje konvencionalnog cementa (cink-fosfatni ili klasični staklenoionomerni cement) samo na rubove nadoknade. U tom slučaju nepovoljno svojstvo - marginalna topivost i posljedični nastanak diskrepancije - kod konvencionalnih cemenata može rezultirati padom retencije cementa nakon određenog vremena $(10,14,15)$.

Na tržǐ̌tu postoje i tehnološki specijalno razvijeni cementi za dugotrajno privremeno cementiranje koji osiguravaju odgovarajuću retenciju nadoknade od 6 mjeseci do godinu dana. Taj način polutrajnog cementiranja i mogućnost uklanjanja obrađena je u nekim studijama $(16-22)$.

$\mathrm{U}$ uvjetima in vitro mogu se simulirati neki čmbenici iz usne šupljine koji mogu utjecati na promjene svojstava dentalnih gradivnih materijala u uvjetima in vivo. Jedan od najčešće korištenih načina u simulaciji umjetnoga starenja jest termocikliranje koje uključuje podvrgavanje eksperimentalnih uzoraka ponovljenim ciklusima niske i visoke temperature kako bi se postigle temperaturne promjene koje se dogadaju u usnoj šupljini (23). Temperaturne promjene induciraju stres u dentalnim materijalima pa njihova svojstva mogu biti promijenjena.

Mehanička ciklička opterećenja metoda su simulacije okluzalnih stresova u usnoj šupljini. Spomenuti način simulacije žvačnih ciklusa korišten je u opservaciji retencijskih sila dentalnih cemenata, promatrano u različitim vremenskim točkama $(24,25)$.

Zato je svrha ove studije bila ispitati utjecaj umjetnoga starenja na retencijsku silu polutrajnih cemenata te je li moguće upotrijebiti konvencionalne cemente za semipermanentno cementiranje, uz specifičnu prilagodbu njihova protokola.

Testirane su sljedeće hipoteze: ne postoji razlika u retencijskim silama testiranih cemenata - GC Fuji Temp LT, Telio CS Cem Implant i Harvard Cement (standardna i prilagođena tehnika cementiranja) promatrano na vremenskim točkama mjerenja; nema utjecaja termocikliranja i mehaničkih cikličkih opterećenja na fizičku retenciju testiranih cemenata promatrano na vremenskim točkama mjerenja.

\section{Materijal i metode}

U studiji je korišteno 40 titanijevih nadogradnji (Easy abatment NP 0,75; Nobel Biocare, Švedska) koničnosti $8^{\circ}$, visine $5,5 \mathrm{~mm}$ i 40 replika implantanta (Implant Replica, Nobel Biocare, Švedska). Abutmenti su pričrvršćeni na implantate analoge silom od $35 \mathrm{~N} / \mathrm{cm}$. Krunice su izrađene od legure Co-Cr-Mo (Bond NF - Nickel free, Interdent, Slovenija) (slike 1. i 2.).

Pristupni kanal nadogradnje zatvoren je privremenim svjetlosnopolimerizujućim kompozitom (Temp it, Spident, Južna Koreja).

Nadoknade su izrađene na osnovi silikonskog kalupa okluzalne dvije trećine krunice akrilatnoga Frasaco zuba drugoga donjeg premolara. Unutarnji aspekt nadoknade i debljina cementnoga prostora standardizirani su upotrebom plastičnih kapica za lijevanje (Plastic Coping Easy Abatment Engaging NP $2 \mathrm{pkg}$ ) visine $7 \mathrm{~mm}$. 

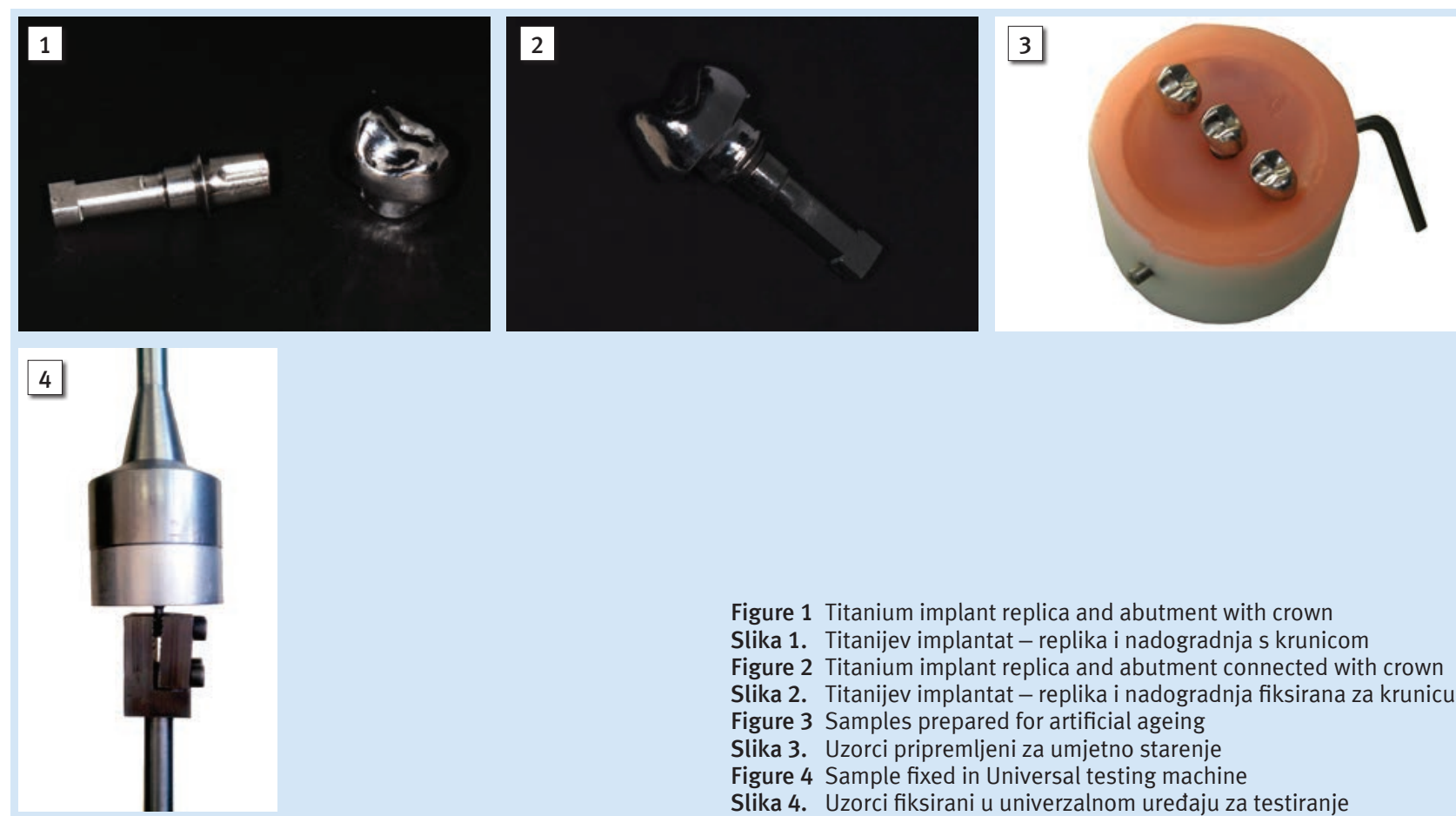

Figure 1 Titanium implant replica and abutment with crown Slika 1. Titanijev implantat - replika i nadogradnja s krunicom Figure 2 Titanium implant replica and abutment connected with crown Slika 2. Titanijev implantat - replika i nadogradnja fiksirana za krunicu Figure 3 Samples prepared for artificial ageing

Slika 3. Uzorci pripremljeni za umjetno starenje

Figure 4 Sample fixed in Universal testing machine

Slika 4. Uzorci fiksirani u univerzalnom uređaju za testiranje

the inner surface was sandblasted with 50 micron aluminumoxide particles. The crown fitting check was completed using silicon material (Fit Checker, GC Co, Tokyo, Japan). The samples were cleaned in an ultrasound bath and with hydrofluoric acid to avoid contamination of the binding surfaces.

The 40 samples were then divided into four groups with 10 samples each. The study evaluated three commercially available cements: semipermanent cement (Telio CS Cem Implant, Ivoclar Vivadent, Liechtenstein), semipermanent cement (GC Fuji Temp LT, GC, Japan) and conventional zinc-phosphate cement for permanent cementation (Harvard Cement, Harvard, Germany) (standard and modified cementing technique) (Table 1).

Semipermanent cements Telio CS Cem Implant and GC Fuji Temp LT, and conventional zinc phosphate Harvard Cement (standard technique) were prepared according to the manufacturer's instructions and applied to the complete in-
Nakon lijevanja je vanjska površina nadoknade visokopolirana, te pjeskarena česticama aluminijeva oksida veličine 50 $\mu \mathrm{m}$. Nalijeganje nadoknada provjereno je upotrebom silikonskog materijala za provjeru nalijeganja (Fit Checker, GC Co, Tokio, Japan). Uzorci su očišćeni u ultrazvučnoj kupki i hidrofluoričnom kiselinom da bi se izbjegla kontaminacija vezivnih površina.

U četiri skupine od po deset uzoraka raspoređeno je 40 uzoraka. U studiji su evaluirana tri komercijalno dostupna cementa - semipermanentni cement (Telio CS Cem Implant, Ivoclar Vivadent, Lihtenštajn), cement za dugotrajno privremeno cementiranje (GC Fuji Temp LT, GC, Japan) i konvencionalni cink-fosfatni cement za trajno cementiranje (Harvard Cement, Harvard, Njemačka) (standardna i prilagođena tehnika cementiranja) (tablica 1.).

Semipermanentni cement Telio CS Cem Implant i GC Fuji Temp LT i konvencionalni cink-fosfatni cement - Har-

\begin{tabular}{|c|c|c|c|}
\hline $\begin{array}{c}\text { Group } \\
\text { Grupe }\end{array}$ & $\begin{array}{c}\text { Number of samples } \\
\text { Broj uzoraka }\end{array}$ & Type of cement $\bullet$ Vrsta cementa & Packing mode $\bullet$ Pakiranje \\
\hline 1 & 10 & $\begin{array}{l}\text { Telio CS Cem, IvoclarVivadent, Liechtenstein • } \\
\text { Lihtenštajn }\end{array}$ & $\begin{array}{l}\text { Dual-curing resin semipermanent cement comes as } \\
\text { two pastes in one syringe. } \bullet \text { Dvostruko polimerizujući } \\
\text { kompozitni semipermanentni cement; proizvodi se u dvije } \\
\text { paste u jednoj brizgalici. }\end{array}$ \\
\hline 2 & 10 & Fuji TEMP LT, GC & $\begin{array}{l}\text { Glass ionomer semipermanent cement comes as two pastes } \\
\text { in one syringe. } \bullet \text { Staklenoionomerni semipermanenti } \\
\text { cement; proizvodi se kao dvije paste u jednoj brizgalici. }\end{array}$ \\
\hline 3 & 10 & $\begin{array}{l}\text { Zinc-phosphate cement, Harvard, Germany } \\
\text { (conventional cementing) • Cink-fosfatni cement, } \\
\text { Harvard, Njemačka (konvencionalno cementiranje) }\end{array}$ & $\begin{array}{l}\text { Conventional permanent cement comes as powder and } \\
\text { liquid. • Konvencionalni trajni cement u obliku praha i } \\
\text { tekućine. }\end{array}$ \\
\hline 4 & 10 & $\begin{array}{l}\text { Zinc-phosphate cement, Harvard Germany (modified } \\
\text { cementing technique) } \bullet \text { Cink-fosfatni cement, Harvard } \\
\text { Njemačka (prilagođena tehnika cementiranja) }\end{array}$ & $\begin{array}{l}\text { Conventional permanent cement, comes powder and } \\
\text { liquid. • Konvencionalni trajni cement; u obliku praha i } \\
\text { tekućine. }\end{array}$ \\
\hline
\end{tabular}


ner walls of the crown. For modified cementing technique, zinc phosphate Harvard cements were used according to the manufacturer's instructions, and were applied with a small brush in a thin film band of $1 \mathrm{~mm}$ to the cervical margin of the inner surface of the copings.

The crowns were carefully placed on the abutments and a $5 \mathrm{~kg}$-controlled force was applied using a hydraulic, digital controlled press. The samples cemented with Telio CS Cem Implant were lightened initially for 3 seconds to obtain a rubbery consistency and for easier removal of excess material. The excess material of the samples with GC Fuji Temp LT and Harvard Cement, Harvard, Germany, was also removed once the cements reached a rubbery consistency. All samples were subjected to controlled pressure for 10 minutes to complete the process of chemical polymerization and set the cement. The samples were stored for the next 24 hours in artificial saliva at $37^{\circ} \mathrm{C}$ temperature, and subjected to a thermocycling process which consisted of 500 cycles of temperature fluctuations $\left(5-55^{\circ} \mathrm{C}\right)$. After that, the samples were fixed in special modules in base of self-curing acrylate material which is similar to the human bone by its modulus of elasticity (Technovit 9100, Heraeus Kultzer, Hanau, Germany) (Figure 3).

Chewing cycle's simulation was performed in masticatory cycle simulating machine (Chewing simulator CS-4.2 economy line (SD Mechatronik, Germany)) in an artificial saliva environment. with a predetermined schedule in term of simulation of mouth function periods (7 days, 3, 6, 9 and 12 months function simulation) (Figure 3, Table 2). vard Cement (standardna tehnika) pripremljeni su prema uputama proizvođača i naneseni na kompletnu površinu unutarnjih zidova nadoknade. Za prilagođenu tehniku cementiranja je cink-fosfatni cement pripremljen u skladu s uputom proizvođača i apliciran četkicom u tankom sloju $1 \mathrm{~mm}$ širine, na cervikalni rub unutarnje površine krunice.

Nadoknade su pažljivo postavljene na nadogradnju i tijekom vezivanja cementa je, $s$ pomoću hidraulične preše $s$ digitalnom kontrolom, primijenjena kontrolirana sila od $5 \mathrm{~kg}$. Uzorci pričvršćeni semipermanentnim cementima prosvjetljeni su inicijalno 3 sekunde kako bi se dobila gumasta konzistencija cementa i olakšalo uklanjanje mogućega viška. Višak cementa na uzorcima pričvršćenima cementima GC Fuji Temp LT i Harvard također je uklonjen kada je cement postigao gumastu konzistenciju. Svi uzorci su pod kontroliranim pritiskom bili 10 minuta kako bi se završio proces vezivanja cemenata. Sljedeća 24 sata uzorci su bili pohranjeni u mediju umjetne pljuvačke na temperaturi od $37^{\circ} \mathrm{C}$ i podvrgnuti procesu termocikliranja koji se sastojao od 500 ciklusa temperaturnih fluktuacija $\left(5-55^{\circ} \mathrm{C}\right)$. Nakon toga su fiksirani u specijalnim modulima u bazu od samovezujućeg akrilata koji je prema svojoj elastičnosti sličan ljudskoj kosti (Technovit 9100, Heraeus Kultzer, Hanau, Njemačka) (slika 3.).

Simulacija žvačnih ciklusa obavljena je u uređaju za simulaciju mastikatornih ciklusa (Chewing simulator CS-4.2 economy line - SD Mechatronik, Njemačka) u mediju umjetne pljuvačke, prema utvrđenom rasporedu simulacije razdoblja funkcije u usnoj šupljini (7 dana, 3, 6, 9 i 12 mjeseci) (tablica 2.). Kada je kompletiran proces umjetnoga starenja, retencij-

Table 2 Testing periods of artificial aging with mechanical loading cycles

Tablica 2. Testna razdoblja umjetnoga starenja s ciklusima mehaničkih cikličkih opterećenja

\begin{tabular}{c|c|c}
$\begin{array}{c}\text { Testing rounds } \bullet \\
\text { Krugovi testiranja }\end{array}$ & $\begin{array}{l}\text { Testing periods } \bullet \\
\text { Testna razdoblja }\end{array}$ & $\begin{array}{c}\text { Number of masticatory cycles (mechanical loading cycles) } \\
\text { Broj žvačnih ciklusa (mehanička ciklička opterećenja) } \\
1\end{array}$ \\
\hline 2 & 3 days $\bullet$ dana & 2500 \\
\hline 3 & 6 months $\bullet$ mjeseca & 5000 \\
\hline 4 & 9 months $\bullet$ mjeseci & 7500 \\
\hline 5 & 12 months $\bullet$ mjeseci & 10000 \\
\hline
\end{tabular}

Once the artificial ageing process of the material was complete, the retention force of the samples was measured with a Universal testing machine (Instron 1122) (Figure 4). Uniaxial tensile force with 1 millimeter per minute speed was applied and the results were recorded on/ the testing machine graph. The samples were cleaned under the same protocol between the individual testing rounds - crowns were subjected to $220^{\circ} \mathrm{C}$ temperature ( 2 cycles with 12 minutes duration) at the sterilization device to enhance degradation of cement residues and to make its removal easier. The samples were then cooled at the room temperature. Cement excess was at first removed by hand tools, while the rest of the cement from rough inner surface of the crown was removed by sand blasting.

After sterilization, the inner surface of crown copings were cleaned by sandblasting with aluminum oxide $50 \mu \mathrm{m}$ particles under the pressure of 2,5 barometers and dried using com- ska sila uzoraka izmjerena je u univerzalnom uređaju za testiranje (Instron 1122) (slika 4.). Aplicirana je uniaksijalna zatezna sila brzine 1 milimetar u minuti, a rezultati su zabilježeni na grafikonu kidalice.

Između pojedinih krugova testiranja uzorci su čišćeni pre$\mathrm{ma}$ istom protokolu, a nadoknade su podvrgnute djelovanju temperature od $220{ }^{\circ} \mathrm{C}$ ( 2 ciklusa po 12 minuta) u uređaju za sterilizaciju da bi se pospješila degradacija ostataka cementa i olakšalo njegovo uklanjanje.

Uzorci su zatim ohlađeni na sobnoj temperaturi. Višak cementa najprije je uklonjen ručnim instrumentima, a ostatak $s$ hrapave unutarnje površine nadoknade očišćen je pjeskarenjem.

Nakon završene sterilizacije, unutarnja površina krunica očišćena je pjeskarenjem $s$ česticama aluminijeva oksida veličine $50 \mu \mathrm{m}$, pod pritiskom od 2,5 bara i krunice su osušene komprimiranim zrakom. Ostatci cementa s površine na- 
pressed air. Cement residues from the abutment surface were hand-removed with plastic instrument and surface of abutment was polished with paste (CleanJoy, Voco, Germany). Abutments and crowns were cleaned by ultrasound in the final phase of the cleaning protocol. After cleaning, the samples were inspected under ten times magnification to ensure that the surfaces of the samples were free of residual cement.

\section{Statistical analysis}

The statistical analyses were performed using the SPSS software (IBM SPSS statistics 24.0, IBM Corporation, New York, United States) at a 5\% significance level. The Anova test and post-hoc tests, the Tukey and Tamhane's T2, were applied to quantitative and continuous variables.

\section{Results}

The highest initial retention force was recorded for zincphosphate cement - conventional cementing $(198.00 \pm 61.90$ $\mathrm{N})$ followed by (in descending order) zinc-phosphate cement - modified cementing technique $(152.00 \pm 45.42 \mathrm{~N})$, cement for temporary long term cementation - Fuji Temp LT $(57,70 \pm 20,40 \mathrm{~N})$ and semipermanent cement - Telio CS Cem Implant (56.10 \pm 18.68 N) (Table 3).

After exposing the samples to artificial aging, a decrease in retention force was recorded for both cementing techniques. After 12 months, the retention value in those samples that were cemented using a conventional technique was 88 . $9 \pm 14.45 \mathrm{~N}$. This value was lower $(48.15 \pm 14.41 \mathrm{~N})$ for the samples cemented by the modified techniques.

Retention values of samples cemented with both techniques (conventional and modified) decreased gradually during all tested periods. A significant difference was not recorded during the initial measurement $(p=0,074)$ but it was found on the $7^{\text {th }}$ day $(p=0,019), 3^{\text {rd }}$ month $(p<0,001), 6^{\text {th }}$ month $(\mathrm{p}=0,005), 9^{\text {th }}$ month $(\mathrm{p}<0,001)$ and $12^{\text {th }}$ month $(\mathrm{p}<0,001)$ (Table 3).

Semipermanent cements Telio CS Cem Implant and long term temporary cement GC Fuji Temp LT also had a similar initial retention force $(56,10 \pm 18,68 \mathrm{~N}$ and $57,70 \pm 20,40 \mathrm{~N})$ without a significant difference $(p=1,000)$.

Subsequent testing rounds recorded a continuous decrease in retention force for both semipermanent cements, but without any statistically significant difference at all tested periods: $7^{\text {th }}$ day $(\mathrm{p}=0,988), 3^{\text {rd }}$ month $(\mathrm{p}=1,000), 6^{\text {th }}$ month $(\mathrm{p}=0,994), 9^{\text {th }}$ month $(\mathrm{p}=0,892)$ and $12^{\text {th }}$ month $(\mathrm{p}=0,997)$ (Table 3).

\section{Discussion}

The factors that determine the retention of the crown on the implant abutment are numerous: height and shape of abutment, crown/abutment fit, surface roughness of crown and abutment, functional ageing, cement film thickness, cement type, and the cementation technique used $(20,22$ 28). The present study evaluated two of these factors: cement type and ageing. dogradnje ručno su uklonjeni plastičnim instrumentima i površina je polirana pastom (CleanJoy, Voco, Njemačka). I nadgradnje i krunice očišćeni su ultrazvučno u finalnoj fazi protokola čišćenja. Nakon toga obavljen je pregled površine pod povećanjem od 10 puta da bismo bili sigurni da na površini uzorka više nema ostataka cementa.

\section{Statistička analiza}

Statistička analiza obavljena je u softveru SPSS (IBM SPSS statistics 24.0, IBM Corporation, New York, SAD) na razini značajnosti od $5 \%$. ANOVA i post-hoc test te Tukeyjev test i Tamhaneov T2 korišteni su za kvantitativne i kontinuirane varijable.

\section{Rezultati}

Najviša inicijalna retencijska sila zabilježena je za cink-fosfatni cement - konvencionalna tehnika cementiranja $(198,00$ $\pm 61,90 \mathrm{~N}$ ) a slijede (u silaznom nizu) cink-fosfatni cement - prilagođena tehnika cementiranja $(152,00 \pm 45,42 \mathrm{~N})$, cement za dugotrajno privremeno cementiranje Fuji Temp LT $(57,70 \pm 20,40 \mathrm{~N})$ i semipermanentni cement Telio CS Cem Implant $(56,10 \pm 18,68 \mathrm{~N})$ (tablica 3.).

Nakon izlaganja uzoraka umjetnom starenju, opadanje retencijske sile zabilježeno je pri primjeni obiju tehnika cementiranja. Nakon 12 mjeseci su retencijske vrijednosti u uzorcima cementiranima konvencionalnom tehnikom iznosile $88,90 \pm 14,45 \mathrm{~N}$. Izmjerena vrijednost bila je niža $(48,15$ $\pm 14,41 \mathrm{~N})$ u uzorcima cementiranima prilagođenom tehnikom.

Retencijske vrijednosti uzoraka cementiranih objema tehnikama (konvencionalnom i prilagođenom) snižavaju se postupno tijekom svih testnih razdoblja.

Tijekom inicijalnog mjerenja nije uočena statistički značajna razlika $(\mathrm{p}=0,074)$, ali je zabilježena u sedmom danu $(\mathrm{p}=0,019)$, trećem mjesecu $(\mathrm{p}<0,001)$, šestom mjesecu $(\mathrm{p}$ $=0,005)$, devetom mjesecu $(\mathrm{p}<0,001)$ i dvanaestom mjesecu $(\mathrm{p}<0,001)$ simulirane funkcije (tablica 3.).

Semipermanentni cement Telio CS Cem Implant i dugotrajni privremeni cement GC Fuji Temp LT također pokazuju slične vrijednosti retencijskih sila $(56,10 \pm 18,68 \mathrm{~N}$ i 57 , $70 \pm 20,40 \mathrm{~N})$ bez statistički značajne razlike $(\mathrm{p}=1,000)$.

U sljedećim ciklusima testiranja zabilježen je kontinuirani pad retencijskih sila za oba semipermanentna cementa, ali bez statistički značajne razlike u svim testnim razdobljima sedam dana $(\mathrm{p}=0,988), 3$ mjeseca $(\mathrm{p}=1,000), 6$ mjeseci $(\mathrm{p}$ $=0,994), 9$ mjeseci $(\mathrm{p}=0,892)$ i 12 mjeseci $(\mathrm{p}=0,997)(\mathrm{ta}-$ blica 3.).

\section{Rasprava}

Mnogobrojni su čimbenici koji determiniraju retenciju krunica na nadogradnjama implantata - visina i oblik nadogradnje, nalijeganje krunica na nadogradnju, površinska hrapavost krunice i nadogradnje, funkcionalno starenje, debljina cementnoga filma, vrsta cementa i upotrijebljena tehnika cementiranja $(20,22$ - 28). Ova studija ispitivala je utjecaj dvaju čimbenika - vrstu cementa i starenje. 
Table 3 Retention force of semipermanent cements and zinc phosphate cemenet (conventional and modified cementing techniques) recorded in $\mathrm{N}$

Tablica 3. Vrijednosti retencijskih sila semipermanentnih cemenata i cink-fosfatnoga cementa (konvencionalna i prilagođena tehnika cementiranja) zabilježena u njutnima (N)

Type of cement $\bullet$ Vrsta cementa

Mean \pm SD

Initial measurement $\bullet$ Inicijalno mjerenje

semipermanent cements $\bullet$ semipermanenti cementi

\begin{tabular}{|c|c|c|}
\hline Telio CS & $56.10 \pm 18.68$ & \multirow{2}{*}{1.000} \\
\hline GC Fuji Temp LT & $57.70 \pm 20.40$ & \\
\hline \multicolumn{3}{|l|}{ zinc phosphate cement $\bullet$ cink-fosfatni cement } \\
\hline conventional cementing technique $\bullet$ konvencionalna tehnika cementiranja & $198.00 \pm 61.90$ & 0.074 \\
\hline
\end{tabular}

7 days $\bullet 7$ dana

semipermanent cements $\bullet$ semipermanenti cementi

Telio CS

GC Fuji Temp LT

zinc phosphate cement $\bullet$ cink-fosfatni cement

conventional cementing technique $\bullet$ konvencionalna tehnika cementiranja

modified cementing technique $\bullet$ prilagođena tehnika cementiranja

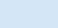

\begin{tabular}{c|c|}
\hline GC Fuji Temp LT & $33.50 \pm 12.71$ \\
\hline zinc phosphate cement $\bullet$ cink-fosfatni cement & $38.35 \pm 14.41$ \\
\hline conventional cementing technique $\bullet$ konvencionalna tehnika cementiranja & $179,30 \pm 53,78$ \\
\hline modified cementing technique $\bullet$ prilagodena tehnika cementiranja & $\mathbf{0 , 0 1 9}$
\end{tabular}

\section{3 months $\bullet 3$ mjeseca}

semipermanent cements $\bullet$ semipermanenti cementi

Telio CS

GC Fuji Temp LT

zinc phosphate cement $\bullet$ cink-fosfatni cement

conventional cementing technique $\bullet$ konvencionalna tehnika cementiranja

modified cementing technique $\bullet$ prilagođena tehnika cementiranja

\begin{tabular}{c|c} 
& \\
\hline $32.10 \pm 16.82$ & 1.000 \\
\hline $31.90 \pm 12.96$ & \\
\hline $153.85 \pm 47.73$ & $<0.001$
\end{tabular}

6 months $\bullet 6$ mjeseci

semipermanent cements $\bullet$ semipermanenti cementi

Telio CS

GC Fuji Temp LT

zinc phosphate cement $\bullet$ cink-fosfatni cement

conventional cementing technique $\bullet$ konvencionalna tehnika cementiranja

modified cementing technique $\bullet$ prilagođena tehnika cementiranja

$87.80 \pm 35.70$

0.994

$25.30 \pm 15.11$

$28.70 \pm 13.57$

$131.7 \pm 41.26$

$84.05 \pm 36.68$

0.005

9 months $\bullet 9$ mjeseci

semipermanent cements $\bullet$ semipermanenti cementi

\begin{tabular}{c|c}
\hline Telio CS & $18.80 \pm 7,18$ \\
\hline GC Fuji Temp LT & $22.80 \pm 6.96$ \\
\hline zinc phosphate cement $\bullet$ cink-fosfatni cement & \\
\hline conventional cementing technique $\bullet$ konvencionalna tehnika cementiranja & $99.00 \pm 14.10$ \\
\hline modified cementing technique $\bullet$ prilagodena tehnika cementiranja & $62.20 \pm 18.29$
\end{tabular}

12 months $\bullet 12$ mjeseci

semipermanent cements $\bullet$ semipermanenti cementi

Telio CS

GC Fuji Temp LT

zinc phosphate cement $\bullet$ cink-fosfatni cement

conventional cementing technique $\bullet$ konvencionalna tehnika cementiranja

modified cementing technique $\bullet$ prilagođena tehnika cementiranja

\begin{tabular}{l|l} 
& \\
$15.55 \pm 5.52$ & \multirow{2}{*}{0.997} \\
$16.55 \pm 3,88$ & \\
\hline & \\
\cline { 1 - 1 } $88.90 \pm 14.45$ & \multirow{2}{*}{$<\mathbf{0 . 0 0 1}$}
\end{tabular}

Bold indicates $\mathrm{p}<.05$

The p values corresponds to statistically difference between 2 semipermanent cements and 2 different cementing techniques with zinc phosphate cement $\bullet$ p vrijednost odgovara statističkoj razlici između 2 semipermanentna cementa i 2 različite tehnike cementiranja s cink-fosfatnim cementom

Initially, the measurements of retention force of samples that were not exposed to artificial ageing showed that the highest retention force was zinc-phosphate cement Harvard, conventional cementing technique $(198.00 \pm 61.90 \mathrm{~N})$ followed by zinc-phosphate cement Harvard - modified cementing technique $(152.00 \pm 45.42 \mathrm{~N})$, temporary cement for long-term temporary cementation - Fuji TEMP LT
Inicijalno mjerenje retencijske sile uzoraka koji nisu bili izloženi umjetnom starenju pokazalo je najvišu vrijednost kod cink-fosfatnoga cementa Harvard - konvencionalna tehnika cementiranja $(198,00 \pm 61,90 \mathrm{~N})$ a slijede cink-fosfatni cement Harvard - prilagođena tehnika cementiranja $(152,00$ $\pm 45,42 \mathrm{~N}$ ), privremeni cement za dugotrajno cementiranje Fuji TEMP LT $(57,70 \pm 20,40 \mathrm{~N})$ i semipermanentni cement 
$(57.70 \pm 20.40 \mathrm{~N})$ and semipermanent cement - Telio CS Cem Implant $(56,10 \pm 18,68 \mathrm{~N})$. Our results are in lines with a study by Lugas et al (21), which evaluated the degree of retrievability of three different cements, ranked from temporary to definitive cementation use.

Nowadays, zinc-phosphate cement is still used in many studies in order to compare conventional and specially developed cements $(14,15,29-33)$. All these studies, including this one, recorded high values of retention for zinc-phosphate cement immediately after cementing.

The high retention force of permanent cements requires the application of significant force during the crown removal from the implant abutment. Due to the need to reduce the load on the implant/abutment/crown complex during the restoration removal and securing the retrievability of the implant-supported crowns, the idea of examining the effects of modifying the cementation technique of conventional cements emerged.

Our study found the difference between the initial retention of unloaded samples, cemented with conventional technique by applying cement to the entire inner surface of the crown $(198,00 \pm 61,90 \mathrm{~N})$ and with the modified technique of applying the cement layer only along the crown's edge $(152,00 \pm 45,42 \mathrm{~N})$. Despite the fall of the retention value of cement during the mouth function, the retention force value still remained high after one year when the conventional technique was utilized $(88.9 \pm 14.45 \mathrm{~N})$. This value was significantly lower for samples cemented using the modified technique $(48.15 \pm 14.41 \mathrm{~N})$.

Although showing extremely high initial retention values, the main deficiency of zinc-phosphate cement appeared - the dissolution of the cement layer on the marginal parts as well as marginal discrepancy, which has been described in previous studies $(10,14,34)$.

Considering that the retention surface is directly proportional to the cement retention value, the modified cementing technique used was applying a $2 \mathrm{~mm}$ wide cement layer only along the crown edges. During the time of the study, our measurements showed that the cement dissolved at the crown edges, which resulted in the retention value weakening gradually. There were significant differences between the retention values of conventional and modified cementing techniques during all the testing intervals of our study $(\mathrm{p}=0,019, \mathrm{p}<0,001, \mathrm{p}=0,005, \mathrm{p}<0,001, \mathrm{p}<0,001)$ except the initial measurement $(p=0,074)$. These findings are in contrast to the results of Mehl et al. and Wolfart et al. studies $(33,35)$.

However, it should be mentioned that the methodology of these studies did not include artificial ageing of the samples. The reason behind the initial retention force value recorded for zinc-phosphate cement used with metal components of implantoprosthetic reconstructions is its proven high bond with crowns, made of both precious and non-precious alloys. Ergin et al. documented the stronger retention force of zinc-phosphate cement on the surface of a non-precious alloy crown (36). For the purpose of this study, the crowns were manufactured of CoCrMo alloy as one of the most frequently used non-precious alloys in modern dentistry, which continues to be the subject of new research on its additional
Telio CS Cem Implant $(56,10 \pm 18,68$ N). Naši rezultati u skladu su s onima iz studije Lugasa i suradnika (21) koji su istraživali stupanj mogućnosti uklanjanja triju različitih cemenata rangiranih od privremenoga do definitivnoga.

Danas se cink-fosfatni cement još uvijek koristi u mnogim studijama za usporedbu konvencionalnih i specijalno dizajniranih cemenata $(14,15,29$ - 33). U svima njima, uključujući i našu studiju, zabilježene su visoke retencijske vrijednosti cink-fosfatnoga cementa neposredno nakon cementiranja.

Visoka retencijska sila trajnih cemenata zahtijeva primjenu znatne sile pri uklanjanju krunice $s$ nadogradnje implantata. U skladu s potrebom reduciranja opterećenja kompleksa implant - nadogradnja - krunica, tijekom uklanjanja nadogradnje i osiguranja mogućnosti uklanjanja krunica postavljenih na implantate, nastala je ideja da se ispita učinak prilagođene tehnike cementiranja kod konvencionalnih cemenata. U našoj studiji pronađena je razlika između inicijalne vrijednosti neopterećenih uzoraka cementiranih konvencionalnom tehnikom pri apliciranju cementa na cijelu unutarnju površinu krunice $(198,00 \pm 61,90 \mathrm{~N})$ i pričvršćenih modificiranom tehnikom kod koje se cementni sloj nanosi samo duž ruba krunice $(152,00 \pm 45,42 \mathrm{~N})$. Unatoč padu vrijednosti retencijske sile cementa tijekom funkcije u usnoj šupljini, i poslije jedne godine još je uvijek visoka ako je korišstena konvencionalna tehnika cementiranja $(88,9 \pm 14,45 \mathrm{~N})$. Ta je vrijednost znatno niža u uzorcima cementiranima prilagođenom tehnikom cementiranja $(48,15 \pm 14,41 \mathrm{~N})$.

Iako pokazuje iznimno visoku inicijalnu retencijsku vrijednost, glavni nedostatak cink-fosfatnoga cementa jest topljenje cementnoga sloja duž rubnoga dijela krunice i marginalna diskrepancija opisana u dosadašnjim studijama (10, 14, 34).

Imajući na umu da je retencijska površina izravno proporcionalna retencijskoj vrijednosti cementa, prilagođena tehnika cementiranja korišstena je samo uz rub krunice u 2-milimetarskome cementnom sloju. Tijekom rada na studiji mjerenja su pokazala da se cement rastapa duž ruba krunice, što rezultira postupnim slabljenjem retencijske vrijednosti. U našoj studiji pronađena je značajna razlika između retencijske vrijednosti konvencionalne i prilagođene tehnike cementiranja tijekom svih testiranja $(p=0,019, p<0,001$, $\mathrm{p}=0,005, \mathrm{p}<0,001, \mathrm{p}<0,001)$, osim pri inicijalnom mjerenju $(\mathrm{p}=0,074)$. Ti su rezultati u suprotnosti $s$ rezultatima iz studija i Mehla i Wolfarta sa suradnicima $(33,35)$. Ipak, potrebno je napomenuti da metodologija tih studija nije uključivala umjetno starenje uzoraka. Razlog koji se nalazi iza inicijalna retencijske sile zabilježene za cink-fosfatni cement upotrijebljen $s$ metalnim komponentama implantoprotetičke rekonstrukcije, jest njegova dokazana visoka sila vezivanja s krunicama od plemenitih i neplemenitih legura. Ergin i suradnici dokumentirali su jaču retencijsku silu cink-fosfatnoga cementa za površinu krunica od neplemenitih legura (36). Za potrebe ove studije krunice su proizvedene od legure Co-Cr-Mo kao jedne od najčešće korištenih neplemenitih slitina u suvremenoj stomatologiji koja nastavlja biti predmetom novih istraživanja zbog svojih dodatnih performansi, što je važno u dizajniranju i proizvodnji ma- 
performance which is important in designing and manufacturing materials with optimal characteristic (37). On the other hand, implant abutments are made of titanium, which accounts for the high initial retention value of zinc phosphate cement immediately after cementing.

The results of this study showed that semipermanent cements Telio CS $(56.10 \pm 18.68 \mathrm{~N})$ and GC Fuji Temp LT $(57.70 \pm 20.40 \mathrm{~N})$ also had a similar initial retention force. The retention force decreased gradually along with all mechanical loading cycles' levels. Comparing the retention force of unloaded samples and retention values measured after 12 months, decreases in retention were recorded $(15.55 \pm 5.52 \mathrm{~N}$ and $16.55 \pm 3.88 \mathrm{~N})$. This still represented a value high enough for enabling crown stability in function, and low enough to allow for the prosthetics reconstruction to be removed from the abutment without trauma. Hence, these cements fulfil the retrievability condition which was the basic idea in their technological development. Our study showed that the decementation forces of both the semipermanent cements which we evaluated depend significantly on ageing levels. These findings were confirmed in the Kappel et al study (20).

The studies conducted by Mundt et al and Alvarez-Arenal et al also concluded that the semipermanent model of cementing provides crown retrievability $(38,39)$. A retrospective study conducted by Schwarz et al. found a high survival rate for both, semipermanent and permanent cementing techniques on implant abutments (16).

The cements examined in our study, Telio CS Implant (dual-curing resin cement) and GC Fuji Temp LT (glass ionomer self-cure cement), are originally made for long-term, temporary cementing of crowns on implant abutments. These cements are highly sensitive to the presence of humidity during the polymerization process. The fact that our study was conducted in a laboratory, where it was possible to maintain absolutely controlled dry conditions, could have significant impact on the retention values we recorded. It indicates the significance of having an adequate and effective cementing protocol in oral mouth conditions where it is difficult to work in the above mentioned conditions. Maintaining the retention value at a level sufficient for enabling crown stability in function, one year after cementation could be explained with its specific glass-ionomer formulation. Conventional glass-ionomer material shows the material expansion during the initial setting time, which reduces the net amount of curing shrinkage, lower degree of shrinkage comparing to resin modified glass-ionomer (40) and tooth like coefficient of thermal expansion. These factors could maintain the marginal seal and slow down the dissolution of cement.

Bearing in mind the fact that the retrievability of implant-supported crowns is an essential requirement of modern cement systems in implantoprosthetics, it is clear why the semipermanent cements were the subject of this study. Semipermanent cements and the modality of semipermanent cementing are still insufficiently tested. Since there are a relatively small number of studies dealing with this issue on the global level, this study contributes significantly to improving knowledge and understanding in this relatively new field of fixed prosthetics. terijala s optimalnim karakteristikama (37). S druge strane, nadogradnje su izrađene od titanija, što rezultira visokom retencijskom vrijednošću cink-fosfatnoga cementa neposredno nakon cementiranja.

Rezultati ove studije pokazuju da semipermanentni cementi Telio CS $(56,10 \pm 18,68 \mathrm{~N})$ i GC Fuji Temp LT $(57,70$ $\pm 20,40 \mathrm{~N})$ također pokazuju slične inicijalne retencijske sile. Retencijska sila slabi postupno tijekom svih razina mehaničkih cikličnih opterećenja. Uspoređujući retencijske sile neopterećenih uzoraka i retencijske vrijednosti izmjerene nakon 12 mjeseci u funkciji, zabilježeno je slabljenje retencije $(15,55 \pm 5,52 \mathrm{~N}$ i $16,55 \pm 3,88 \mathrm{~N})$. Ona ima i dalje dovoljno visoku vrijednost da osigura stabilnost krunice u funkciji, ali i dovoljno nisku da bez traume omogući uklanjanje protetičke nadoknade s nadogradnje. Zato ti cementi ispunjavaju uvjete o mogućnosti njihova uklanjanja, što je bila osnovna ideja u njihovu tehnološkom razvoju. Ova studija pokazuje da sila decementiranja obaju ispitivanih semipermanentnih cemenata ovisi uglavnom o razini starenja. Ovi su rezultati potvrđeni u studiji Kappela i suradnika (20).

U studijama koje su proveli Mundt i Alvarez sa suradnicima također se ističe da semipermanentni način cementiranja osigurava mogućnost uklanjanja krunica $(38,39)$. U retrospektivnoj studiji Schwarza i suradnika zabilježen je visok stupanj preživljavanja za obje tehnike cementiranja - i semipermantnu i permanentnu - na nadogradnju implantata. (16).

Cementi ispitivani u našoj studiji - Telio CS Implant (dvostruko polimerizujući kompozitni cement) i GC Fuji Temp LT (staklenoionomerni samovezujući cement) izvorno su proizvedeni za dugotrajno, privremeno cementiranje krunica na nadogradnjama implantata. Visoko su osjetljivi na vlagu tijekom procesa polimerizacije. Činjenica da je ova studija provedena u laboratorijskim uvjetima u kojima se mogla osigurati apsolutna kontrola suhe sredine može znatno utjecati na zabilježenu retencijsku vrijednost. To ističe značenje osiguravanja adekvatnih i efektivnih protokola cementiranja u uvjetima usne šupljine gdje je teško osigurati spomenute uvjete. Održavanje retencijske vrijednosti na razini dovoljnoj za osiguravanje stabilnosti krunice u funkciij godinu dana nakon cementiranja, može se objasniti specifičnom formulacijom staklenoionomernoga cementa.

Konvencionalni staklenoionomerni materijali pokazuju ekspanziju materijala tijekom početnog vezivanja, što smanjuje skupljanje pri polimerizaciji, s nižim stupnjem skupljanja u usporedbi sa staklenoionomerom modificiranim smolom (40) i koeficijent toplinske ekspanzije sličan zubu. Ti čimbenici mogu osigurati rubno zatvaranje i usporiti otapanje cementa.

Imajući na umu činjenicu da je mogućnost uklanjanja krunica postavljenih na implantate veoma važan zahtjev za suvremene cementne sustave u implantoprotetici, jasno je zašto su semipermanentni cementi bili predmet ovog istraživanja. Semipermanentni cementi i način polutrajnog cementiranja još uvijek su nedovoljno ispitani. Budući da je, na globalnoj razini, razmjerno malo studija koje se bave tom problematikom, ova studija znatno pridonosi unaprjeđenju znanja i razumijevanja problematike u ovom razmjerno novom području fiksne protetike. 
The limitations of this study should be mentioned. To the best of authors knowledge, there is a lack of studies concerning semipermanent cements. Retention for semipermanent cements was tested only up to 1 year of function where the last measured level showed clinically acceptable retention. Therefore, further research should focus on increasing the quantitative level of artificial ageing to test the ultimate functional limits of these cements. Since the results were obtained in an in vitro study, clinical research is needed to confirm these findings.

Thermal cycling is the most widely used testing procedure of arteficial ageing of dental materials. A wide variation in thermal cycling parameters applied in experimental studies has been identified and there is an apparent lack of a standardized protocol evident from comparison across different studies (23). In the current study, thermal cycling procedures were performed in accordance to ISO standard ISO/TR 11405:1994(E). An identical, or similar protocol, has been used in other studies $(24,25,27,41)$.

A great variation in the numbers of cycles that equate to average human daily, weekly and yearly masticatory function could be registered. Due to heterogeneity of data regarding loading conditions - number of cycles, force intensity and testing chamber medium condition, it is difficult to make a comparation between diferent studies $(24,25,34,39)$. Cyclic loading tests require more standardized guidelines for testing and reporting.

As reference conditions for compressive cycling loading, the current study used the values from the study by Dudley et al., whose authors based their reference values on a large study conducted by Graf et al. $(24,25,42)$.

In an effort to create conditions as close as possible to those in the oral cavity, the test was conducted in an artificial saliva environment. The saliva composition used in this study was used according to the study of Huang at all. (43).

Since most of the residual cement remained on the inner surface of the copings, due to its roughness, the cleaning of the copings was more complicated since it additionally included sandblasting. Furthermore, it showed individual characteristics due to different cements that were used in this study. As expected, more residual cement was registered in the samples cemented with Harvard cement - conventional cementation compared to the modified technique, while the residual particles amount of both semipermanent cements after the cleaning procedure was negligible. In order to ensure the samples surface were free of residual cement, the samples were inspected under ten times magnification.

Numerous studies indicate that the value of cement retention increases with increasing surface roughness of the restoration or abutment (44-46). Therefore, in further research, the potential increase in roughness of the bonding surfaces caused by samples sandblasting cleaning procedure and its influence on the retention value of the tested cements must be taken into account.
Potrebno je spomenuti i ograničenja ove studije. Prema saznanjima autora, nema dovoljno istraživanja o polutrajnim cementima. Retencija za polutrajne cemente ispitana je samo do prve godine u funkciji pa je posljednja izmjerena razina pokazala još uvijek klinički prihvatljivu retenciju.

Zato buduća istraživanja treba usmjeriti na povećanje kvantitativne razine umjetnoga starenja kako bi se testirala krajnja ograničenja tih cemenata. $S$ obzirom na to da su rezultati dobiveni u studijama in vitro, potrebne su kliničke studije da bi se oni potvrdili.

Termalno cikliranje najčešće je korišten postupak umjetnoga starenja dentalnih materijala. Utvrđene su velike varijacije u parametrima termalnoga cikliranja, poslije primjene $\mathrm{u}$ eksperimentalnim studijama, a nedostatak standardiziranoga protokola evidentan je iz usporedbe različitih studija. (23). U ovoj studiji primijenjena je procedura termalnog cikliranja u skladu sa standardom ISO ISO/TR 11405:1994 (E). Identičan ili sličan protokol korišsten je u drugim studijama (24, 25, 27, 41).

Zabilježene su velike varijacije u broju ciklusa koji odgovaraju prosječnoj ljudskoj dnevnoj, tjednoj i godišnjoj žvačnoj funkciji. Zbog heterogenosti podataka o uvjetima opterećenja - broju ciklusa, intenzitetu sile i uvjetima medija u komori za testiranje - teško je uspoređivati različite studije $(24,25,34,39)$. Testovi mehaničkih cikličnih opterećenja zahtijevaju standardizaciju smjernice za testiranje i izvještavanje.

Kao referentnu vrijednost za uvjete mehaničkih cikličkih opterećenja, ova studija koristila se vrijednostima iz istraživanja Dudleyja i suradnika koji su bazirali svoje referentne vrijednosti na velikoj studiji Grafa i suradnika $(24,25,42)$.

U nastojanju da se stvore uvjeti što sličniji onima iz usne šupljine, test je obavljen u mediju umjetne pljuvačke. Sastav pljuvačke, upotrijebljene u ovoj studiji, korišsten je prema studiji Huanga i suradnika (43).

Kako je najveći dio rezidualnog cementa preostao na unutarnjoj površini krune, zbog njezine hrapavosti čišćenje je bilo kompliciranije i uključivalo je pjeskarenje. Dodatno, čišćenje je pokazivalo posebna svojstva vezana za različite cemente korištene u studiji. Očekivano, više rezidualnog cementa zabilježeno je na uzorcima pričrršćenima cementom Harvard - konvencionalno cementiranje u usporedbi s prilagođenim, a količina obaju zaostalih semipermanentnih cemenata, poslije završenog protokola čišćenja, bila je zanemariva. Da bismo bili sigurni da na površini uzoraka nisu ostali djelići rezidualnog cementa, uzorci su kontrolirani pod povećanjem od 10 puta.

U mnogobrojnim studijama dokazano je da vrijednost retencijske sile cementa raste $s$ porastom hrapavosti površine nadoknade ili nadogradnje ( 44 - 46). Zato bi se u budućim istraživanjima trebao uzeti u obzir potencijalni porast hrapavosti vezivne površine prouzročen postupkom pjeskiranja uzoraka tijekom čišćenja i njegov utjecaj na retencijsku vrijednost testiranih cemenata. 


\section{Conclusions}

Within the limitations of the present study we can conclude the following: There is a difference in the retention provided by GC Fuji Temp LT, Telio CS Cem Implant and Harvard Cement (standard and modified cementing technique). Thermal cycling and compressive cyclic loading affects the physical retention of tested cements at measurement time points.

The results suggest that the modified cementing technique for zinc-phosphate cement and original semipermanent cements can be recommended for conditional permanent cementing of implant-supported crowns since it still allows retrievability. Retrievability is in direct relation with cement type.

\section{Acknowledgements}

The authors acknowledge and thank the Ministry of Science and Technology of the Republic of Srpska (Bosnia and Herzegovina) for supporting this study. The authors also thank Anita Marie Selec Marie, MSc from Faculty of Philology for the assistance with language and her critical revision.

\section{Conflict of interest}

The authors declare that they have no conflict of interest.

\section{Funding}

The work was supported by Ministry of Science and Technology of the Republic of Srpska (Bosnia and Herzegovina).

Author's contribution: V. V. - coordinated the whole research, participated in testing sample collection, did the statistics and wrote the article; S.M. - design of the study, sample collections, writing article; Z.T. - design of the study, review of the manuscript; N.T. - creating the samples, reviewed selected scientific literature; O.D. - analyzed the data obtained by measurement; T.A. - participated in preparation of individual samples for analysis; R.A. - participated in preparation of individual samples for analysis; M.Š. - second supervisor, participation in laboratory testing; A.T. - first supervisor, suggestions, design of study, review

\section{Zaključak}

Uz postojeća ograničenja studije, možemo zaključiti sljedeće: Postoji razlika u retenciji koju omogućuje GC Fuji Temp LT, Telio CS Cem Implant i Harvard Cement (standardna i prilagođena tehnika cementiranja). Termalni ciklusi i mehanička ciklična opterećenja utječu na fizičku retenciju testiranih cemenata na svim ispitivanim vremenskim točkama. Rezultati pokazuju da se prilagođena tehnika cementiranja cink-fosfatnim cementom i izvornim semipermanentnim cementima može preporučiti za uvjetno trajno cementiranje krunica postavljenih na implantate, jer je osigurana mogućnost njihova uklanjanja. To je svojstvo u izravnom odnosu $s$ vrstom cementa.

\section{Zahvala}

Autori zahvaljuju Ministarstvu znanosti i tehnologije Republike Srpske (Bosna i Hercegovina) na potpori istraživanju. Također zahvaljuju mr. sc. Aniti Marie Selec Marie s Filološkog fakulteta na pomoći u vezi s engleskim jezikom i na njezinoj kritičkoj reviziji.

\section{Sukob interesa}

Autori nisu bili u sukobu interesa.

\section{Financiranje studije}

Studija je potpomognuta od Ministrastva znanosti i tehnologije Republike Srpske, Bosna i Hercegovina

Doprinos autora: V. V. - koordinacija cijelog istraživanja, priprema uzoraka i statistička analiza te pisanje članka; S. M. - dizajn studije, priprema uzoraka, pisanje članka; Z. T. - dizajn studije, revizija članka; N. T. - izrada uzoraka, revizija izabrane znanstvene literature; 0. D. - analiza podataka dobivenih mjerenjima; T. A. - priprema pojedinačnih uzoraka za analizu; R. A. - priprema pojedinačnih uzoraka za analizu; M. Š. - drugi supervizor, sudjeluje u laboratorijskom testiranju; A. T. - prvi supervizor, sugestije, dizajn studije, revizija

\section{Sažetak}

Cilj: Željelo se ispitati kakav je utjecaj umjetnoga starenja na retencijsku silu originalnih (polutrajnih) semipermanentnih cemenata i mogućnost upotrebe konvencionalnih cemenata za polutrajno pričvršćivanje, uz odgovarajuću prilagodbu protokola cementiranja. Materijal i metode: U četiri skupine raspoređeno je 40 krunica izrađenih od Co-Cr-Mo legure (svaka skupina $n=10$ ) i fiksiranih dvama polutrajnim cementima (cementi na bazi smola i staklenoionomera) i jednim konvencionalnim (cinkfosfatni cement), učvršćene konvencionalnom i prilagođenom tehnikom cementiranja na titanijevim nadogradnjama (engl. abutment). Uzorci su bili 24 sata pohranjeni u vlažnim uvjetima na temperaturi od $37^{\circ} \mathrm{C}$ nakon čega su podvrgnuti termocikliranju (500 ciklusa) i mehaničkim cikličnim opterećenjima (simulacija 7 dana, 1, 3, 6, 9 i 12 mjeseci u funkciji). Krunice su uklonjene i zabilježena je retencijska sila. Rezultati: Najvišu inicijalnu retencijsku silu pokazao je cink-fosfatni cement - konvencionalno cementiranje $(198,00 \pm 61,90 \mathrm{~N})$, a slijede ga u silaznom nizu cink-fosfatni cement - prilagođena tehnika cementiranja $(152,00 \pm 45,42 \mathrm{~N})$, dugotrajni privremeni cement - GC Fuji LT (57,70 $\pm 20,40$ $\mathrm{N})$ i semipermanentni cement Telio CS $(56,10 \pm 18,68 \mathrm{~N})$. Poslije 12 mjeseci najviša retencijska sila izmjerena je za cink-fosfatni cement - konvencionalno cementiranje $(88,90 \pm 14,45 \mathrm{~N})$, a slijede cinkfosfatni cement - prilagođena tehnika cementiranja $(48,15 \pm 14,41 \mathrm{~N})$ te semipermanentni cementi GC Fuji Temp LT $(16,55 \pm 3,88 \mathrm{~N})$ i Telio CS Cem Implant $(15,55 \pm 5,52 \mathrm{~N})$. Zaključak: Cink-fosfatni cement - prilagođena tehnika cementiranja i originalni polutrajni cementi (semipermanentni) mogu se preporučiti za uvjetno trajno cementiranje krunica postavljenih na implantate jer omogućuje optimalnu mogućnost uklanjanja krunica postavljenih na implantate.
Zaprimljen: 19. svibnja 2021. Prihvaćen: 3. rujna, 2021.

Adresa za dopisivanje izv. prof. dr. sc. Valentina Veselinović, dr. med. dent.

Sveučilište Banja Luka Medicinski fakultet Katedra za protetiku Save Mrkalja 14

78000 Banja Luka, Bosna i Hercegovina

tel: +38751234100 valentina.veselinovic@med.unibl.org

MeSH terms: stomatološki cementi; testovi mehaničkih svojstava; cementiranje; dentalna implantacija Autorske ključne riječi: mogućnost uklanjanja, semipermanentni cementi; konvencionalni cementi; umjetno starenje; retencijska sila 


\section{References}

1. Hamed MT, Abdullah Mously H, Khalid Alamoudi S, Hossam Hashem AB, Hussein Naguib G. (2020). A Systematic Review of Screw versus Cement-Retained Fixed Implant Supported Reconstructions. Clin Cosmet Investig Dent. 2020 Jan 14;12:9-16.

2. Wittneben JG, Joda T, Weber HP, Brägger U. Screw retained vs. cement retained implant-supported fixed dental prosthesis. Periodontol 2000. 2017 Feb;73(1):141-151.

3. Shi JY, Jie-Ni, Zhuang LF, Zhang XM, Fan LF, Lai HC. Peri-implant conditions and marginal bone loss around cemented and screwretained single implant crowns in posterior regions: A retrospective cohort study with up to 4 years follow-up. PLoS One. 2018 Feb 5;13(2):e0191717

4. Ragauskaitè A, Žekonis G, Žilinskas J, Gleiznys A, Ivanauskienė E, Gleiznys D. The comparison of cement- and screw-retained crowns from technical and biological points of view. Stomatologija. 2017;19(2):44-50.

5. Menini M, Dellepiane E, Pera P, Bevilacqua M, Pesce P, Pera F, Tealdo T. A Luting Technique for Passive Fit of Implant-Supported Fixed Dentures. J Prosthodont. 2016 Jan;25(1):77-82.

6. Karl M, Taylor TD. Bone Adaptation Induced by Non-Passively Fitting Implant Superstructures: A Randomized Clinical Trial. Int J Oral Maxillofac Implants. Mar-Apr 2016;31(2):369-75.

7. Longoni S, Sartori M, Maroni I, Baldoni M. Intraoral luting: modified prosthetic design to achieve passivity, precision of fit, and esthetics for a cement-retained, implant-supported metal-resinfixed complete denture. J Prosthodont. 2010 Feb;19(2):166-70.

8. Spazzin AO, Spazzin WO, Schuh C, Bacchi A, Tosta VB, Marcaccini AM. Technique of framework cemented on prepared abutments to obtain passive fit at fixed complete denture: a 2-year follow-up report. Braz Dent J. Nov-Dec 2014;25(6):565-70.

9. Rosentritt M, Schneider-Feyrer S, Behr M, Preis V. In Vitro Shock Absorption Tests on Implant-Supported Crowns: Influence of Crown Materials and Luting Agents. Int J Oral Maxillofac Implants. January/February 2018;33(1):116-122.

10. Almehmadi N, Kutkut A, Al-Sabbagh M. What is the Best Available Luting Agent for Implant Prosthesis? Dent Clin North Am. 2019 Jul;63(3):531-545.

11. Ozkir SE, Unal SM, Yurekli E, Güven S. Effects of crown retrieval on implants and the surrounding bone: a finite element analysis. J Adv Prosthodont. 2016 Apr;8(2):131-6.

12. Ulrich R, Mühlbradt L, Möhlmann H, Schmid H, Hoss A. Qualitative mechanoperception of natural teeth and endosseous implants. Int J Oral Maxillofac Implants. 1993;8(2):173-8.

13. Bresciano M, Schierano G, Manzella C, Screti A, Bignardi C, Preti G. Retention of luting agents on implant abutments of different height and taper. Clin Oral Implants Res. 2005 Oct;16(5):594-8.

14. Rosas J, Mayta-Tovalino F, Guerrero ME, Tinedo-López PL, Delgado C, Ccahuana-Vasquez VZ. Marginal Discrepancy of Cast Copings to Abutments with Three Different Luting Agents. Int J Dent. 2019 Sep 2;2019:8657582.

15. Saleh M, Ta ar-Faruk S. Comparing the marginal leakage and retention of implant-supported restorations cemented by four different dental cements. Clin Implant Dent Relat Res. 2019 Dec;21(6):1181-1188.

16. Schwarz S, Schröder C, Corcodel N, Hassel AJ, Rammelsberg P. Retrospective comparison of semipermanent and permanent cementation of implant-supported single crowns and FDPs with regard to the incidence of survival and complications. Clin Implant Dent Relat Res. 2012 May;14 Suppl 1:e151-8.

17. Błaszczyszyn A, Kubasiewicz-Ross P, Gedrange T, Dominiak M. Influence of semipermanent cement application used in immediately loaded, implant-supported restorations on crestal bone resorption. Ann Acad Med Stetin. 2013;59(1):66-75.

18. Mehl C, Ali S, El Bahra S, Harder S, Vollrath O, Kern M. Is There a Correlation Between Tensile Strength and Retrievability of Cemented Implant-Retained Crowns Using Artificial Aging? Int J Prosthodont. Jan-Feb 2016;29(1):83-90.

19. Lennartz A, Dohmen A, Bishti S, Fischer H, Wolfart S. Retrievability of implant-supported zirconia restorations cemented on zirconia abutments. J Prosthet Dent. 2018 Nov;120(5):740-746.

20. Kappel S, Chepura T, Schmitter M, Rammelsberg P, Rues S. Effects of Cement, Abutment Surface Pretreatment, and Artificial Aging on the Force Required to Detach Cantilever Fixed Dental Prostheses from Dental Implants. Int J Prosthodont. November/ December 2017;30(6):545-552.
21. Lugas AT, Terzini M, Zanetti EM, Schierano G, Manzella C, Baldi D, et al. In Vitro Impact Testing to Simulate Implant-Supported Prosthesis Retrievability in Clinical Practice: Influence of Cement and Abutment Geometry. Materials (Basel). 2020 Apr 9;13(7):1749.

22. Lugas AT, Terzini M, Zanetti EM, Schierano G, Manzella C, Baldi D, et al. In Vitro Simulation of Dental Implant Bridges Removal: Influence of Luting Agent and Abutments Geometry on Retrievability. Materials (Basel). 2020 Jun 21;13(12):2797.

23. Morresi AL, D’Amario M, Capogreco M, Gatto R, Marzo G, D’Arcangelo C, Monaco A. Thermal cycling for restorative materials: does a standardized protocol exist in laboratory testing? A literature review. J Mech Behav Biomed Mater. 2014 Jan;29:295-308.

24. Dudley JE, Richards LC, Abbott JR. Retention of cast crown copings cemented to implant abutments. Aust Dent J. 2008;53(4):332-9.

25. Dudley J. Influence of compressive cyclic loading on the retention of cast crown copings cemented to implant abutments, doctor thesis, University of Adelaide, 2008.

26. Lopes ACO, Machado CM, Bonjardim LR, Bergamo ETP, Ramalho IS, Witek L, Coelho PG, Bonfante EA. The Effect of CAD/CAM Crown Material and Cement Type on Retention to Implant Abutments. J Prosthodont. 2019 Feb;28(2):e552-e556.

27. Alaa Abou-Obaid, Reem Al-Khudairy. Effect of Abutment Height and Cement Thickness on the Retention of Cement-Retained ImplantSupported Restorations. Adv Dent \& Oral Health. 2018;9(4):555767.

28. Canullo L, Cocchetto R, Marinotti F, Oltra DP, Diago MP, Loi I. Clinical evaluation of an improved cementation technique for implant-supported restorations: a randomized controlled trial. Clin Oral Implants Res. 2016 Dec;27(12):1492-1499.

29. Sheets JL, Wilcox C, Wilwerding T. Cement selection for cementretained crown technique with dental implants. J Prosthodont. 2008 Feb;17(2):92-96.

30. Naumova EA, Roth F, Geis B, Baulig C, Arnold WH, Piwowarczyk A. Influence of Luting Materials on the Retention of Cemented Implant-Supported Crowns: An In Vitro Study. Materials (Basel). 2018 Sep 28;11(10):1853.

31. Kapoor R, Singh K, Kaur S, Arora A. Retention of Implant Supported Metal Crowns Cemented with Different Luting Agents: A Comparative Invitro Study. J Clin Diagn Res. 2016 Apr;10(4):ZC61-4.

32. Montenegro AC, Machado AN, Depes Gouvêa CV. Tensile strength of cementing agents on the CeraOne system of dental prosthesis on implants. Implant Dent. 2008 Dec;17(4):451-60.

33. Mehl C, Harder S, Wolfart M, Kern M, Wolfart S. Retrievability of implant-retained crowns following cementation. Clin Oral Implants Res. 2008 Dec;19(12):1304-11.

34. Pan YH, Ramp LC, Lin CK, Liu PR. Comparison of 7 luting protocols and their effect on the retention and marginal leakage of a cement-retained dental implant restoration. Int J Oral Maxillofac Implants. 2006;21(4):587-592.

35. Wolfart M, Wolfart S, Kern M. Retention forces and seating discrepancies of implant-retained castings after cementation. Int Oral Maxillofac Implants. 2006;21(4):519-525.

36. Ergin S, Gemalmaz D. Retentive properties of five different luting cements on base and noble metal copings. J Prosthet Dent. 2002 Nov;88(5):491-7.

37. Tălu Ş, Stach S, Klaić B, Čelebić A. Evaluation of Topographical Co-Cr-Mo Alloy Surface Changes After Various Finishing Treatments. Acta Stomatol Croat. 2019;53(3):264-273.

38. Mundt T, Heinemann F, Schwahn C, Biffar R. Retrievable, tooth implant-supported, complete-arch fixed restorations in the maxilla: a 6-year retrospective study. Biomed Tech (Berl). 2012 Feb;57(1):39-43.

39. Alvarez-Arenal A, Gonzalez-Gonzalez I, deLlanos-Lanchares $\mathrm{H}$, Brizuela-Velasco A, Ellacuria-Echebarria J. The selection criteria of temporary or permanent luting agents in implant-supported prostheses: in vitro study. J Adv Prosthodont. 2016 Apr;8(2):144-9.

40. Spajić J, Prskalo K, Šariri K, Par M, Pandurić V, Demoli N. Dimensional Changes of Glass lonomers and a Giomer during the Setting Time. Acta Stomatol Croat. 2018;52(4):298-306.

41. Gumus HO, Kurtulus IL, Kuru E. Evaluation and comparison of the film thicknesses of six temporary cements before and after thermal cycling. Niger J Clin Pract. 2018 Dec;21(12):1656-1661.

42. Graf H, Grassel H, Aeberhard HJ. A method for the measurement of occlusal forces in three directions. Helv Odontol Acta 1974;18:711. 
43. Huang HH, Chiu YH, Lee TH, Wu SC, Yang HW, Su KH, Hsu CC. Ion release from $\mathrm{NiTi}$ orthodontic wires in artificial saliva with various acidities. Biomaterials. 2003 Sep;24(20):3585-92.

44. Song MY, An H, Park EJ. The Effect of Temporary Cement Cleaning Methods on the Retention of Crowns. J Prosthodont. 2019 Jan;28(1):e210-e215.

45. Bergamo E, Zahoui A, Luri Amorin Ikejiri L, Marun M, Peixoto da Silva K, G Coelho P, Soares S, A Bonfante E. Retention of zirconia crowns to Ti-base abutments: effect of luting protocol, abutment treatment and autoclave sterilization. J Prosthodont Res. 2021 Jun 30;65(2):171-175

46. Cano-Batalla J, Soliva-Garriga J, Campillo-Funollet M, Munoz-Viveros CA, Giner-Tarrida L. Influence of abutment height and surface roughness on in vitro retention of three luting agents. Int J Oral Maxillofac Implants. Jan-Feb 2012;27(1):36-41. 